\title{
Assistive Conversational Agent for Health Coaching: A Validation Study
}

\author{
Ahmed Fadhil, ${ }^{1}$ Yunlong Wang, ${ }^{2}$ Harald Reiterer ${ }^{2}$ \\ 1 University of Trento, Trento, Italy \\ 2 HCI Group, University of Konstanz, Konstanz, Germany
}

\begin{abstract}
Objective Poor lifestyle represents a health risk factor and is the leading cause of morbidity and chronic conditions. The impact of poor lifestyle can be significantly altered by individual's behavioral modification. Although there are abundant lifestyle promotion applications and tools, they are still limited in providing tailored social support that goes beyond their predefined functionalities. In addition, virtual coaching approaches are still unable to handle user emotional needs. Our approach presents a human-virtual agent mediated system that leverages the conversational agent to handle menial caregiver's works by engaging users (e.g., patients) in a conversation with the conversational agent. The dialog used a natural conversation to interact with users, delivered by the conversational agent and handled with a finite state machine automaton. Our research differs from existing approaches that replace a human coach with a fully automated assistant on user support. The methodology allows users to interact with the technology and access health-related interventions. To assist physicians, the conversational agent gives weighting to user's adherence, based on prior defined conditions.
\end{abstract}

Materials and Methods This article describes the design and validation of CoachAI, a conversational agent-assisted health coaching system to support health intervention delivery to individuals or groups. CoachAI instantiates a text-based health care conversational agent system that bridges the remote human coach and the users.

Results We will discuss our approach and highlight the outcome of a 1-month validation study on physical activity, healthy diet, and stress coping. The study validates technology aspects of our human-virtual agent mediated health coaching system. We present the intervention settings and findings from the study. In addition, we present some user-experience validation results gathered during or after the experimentation.

Conclusions The study provided a set of dimensions when building a human-conversational agent powered health intervention tool. The results provided interesting insights when using human-conversational agent mediated approach in health coaching systems. The findings revealed that users who were highly engaged were also more adherent to conversational-agent activities. This research made key contributions to the literature on techniques in providing social, yet tailored health coaching support: (1) identifying habitual patterns to understand user preferences; (2) the role of a conversational agent in delivering health promoting microactivities; (3) building the technology while adhering to individuals' daily messaging routine; and (4) a socio-technical system that fits with the role of conversational agent as an assistive component.

Future Work Future improvements will consider building the activity recommender based on users' interaction data and integrating users' dietary pattern and emotional wellbeing into the initial user clustering by leveraging information and communication technology approaches (e.g., machine learning). We will integrate a sentiment analysis capability to gather further data about individuals and report these data to the caregiver.

\section{Keywords}

Health coaching - conversational agent - validation study - physical activity - healthy diet - mental wellness 


\section{Introduction}

A varied diet and regular physical activity have significant benefits for individuals' overall health.[1] [2] [3] Similarly, mental wellness is associated with social competence and coping skills that lead to positive outcomes in adulthood and later stages of individuals' life.[4] [5] Although there is benefit of pursuing a healthy lifestyle, several barriers exist in the process of health promotion. For instance, individuals' motivation to change, their demographics and preparedness are all factors that contribute to their intention to follow a healthy lifestyle. Several studies tackled the issue of poor lifestyle through mobile technologies. Mobile applications have been developed via different approaches[6] [7] to mitigate the risk of poor diet, sedentary lifestyle, and anxiety. That said, the learning curve associated with mobile apps is still an issue, especially for individuals with low digital literacy. According to Bosomworth,[8] smartphone users download few apps per month, among which fewer apps are used daily and often abandoned after some time. Other approaches integrated wearable trackers into individuals' daily life to track caloric consumptions, steps taken, and sleeping pattern.[9] However, wearable bands suffer from user abandonment in a short or long term.[10]

The current rise in messaging applications provided another trend to engage individuals into self-tracking their health-related interventions through a conversation medium. Text-based communication is a potentially powerful tool for behavior change, because it is widely available, inexpensive, and easy to use.[11] The rise in artificial intelligence gave birth to messaging applications that run a conversational agent to handle some tasks. Such applications are already used in diet management,[12] [13] [14] physical activity promotion,[15] medication adherence,[16] and mental wellbeing promotion.[17] In addition, users, including the elderly, are familiar with messaging applications due to their low associated learning curve.

This article presents CoachAI, a health intervention delivery coaching web application powered by a conversational agent and a supervised machine-learning model. The machine-learning model performs user clustering based on their physical exercise level. The model is based on real users' data provided by a health care clinic. The platform assists the human agent with user condition tracking, provides the caregiver with insights about their users, and helps track suitable user activities. The approach focuses on providing tailored health activities that adhere to users' preferences. This is achieved by the humanconversational agent mediated coaching which provides a structured health plan to users after analyzing their conversation data with the conversational agent. The bot agent performs data collection, reminders, follow-up by engaging users in prebuilt scripts, and conversations. We believe the conversational agent should prove to be a valuable complement to the human agent, but not a substitute. We describe the results from our validation study to assess the acceptability of this form of coaching by the users, and the feasibility of delivering behavior counseling using the conversational agent medium. Specifically, we are interested in assessing user trust and general satisfaction with the virtual agent to guide our next steps in development and help form hypotheses for future research. Our research work aims at answering the following research questions:

- RQ1: What is the overall user experience of using a CoachAI system?

- RQ2: Will user experience change over time when using CoachAI over 4 weeks?

- RQ3: How much do users prefer a direct human agent support or to what extent does the conversational agent satisfy their needs?

\section{Related Work}

Promoting individuals' behavior has been shown to be a challenging task.[18] [19] To ease this burden while improving the care process, Ibrahim et al[20] developed a multiagent platform to automate the process of collecting user-provided clinical outcome measures without clinician intervention. Health coaching approaches are widely adopted in various health domains to monitor cardiac rehabilitation,[21] promote physical activity at home for elderly,[22] medication adherence,[16] assist pregnant women,[23] [24] promote healthy diet,[12] [13] [14] support individuals with spinal cord injury,[25] and assist with hand therapy.[26] Health coaching systems may vary in their techniques to tackle health issues. That said, most provide remote monitoring to users through either a smartphone, sensors, or wearable trackers. Health coaching is either fully automated (virtual agent), fully manual (human agent), or semi-automated (a combination of the two). Some coaching systems can be text-based,[12] whereas others use short message service (SMS),[27] an avatar or speech-powered agents.[28]

Despite the abundance of health tracking and monitoring systems, tailored coaching and personalized feedback techniques are still in their infancy. A study by Villalonga et al[29] presented an ontology-based approach to model tailored motivational messages for physical activity promotion. The ontology messages can be categorized into multiple classes, e.g., sedentary, mild, or vigorous activities. Similarly, Boratto et al[30] provided an overview of an e-coaching system designed for runners. The platform stimulates individuals' motivation to exercise provided through the coach-user interaction engagement. The 
results showed users' tendency to be more engaged to train when their trainings are developed and remotely supervised by a human coach. The findings showed that e-coaching systems may benefit from considering the support of qualified professionals. The role of mobile apps to facilitate behavior change has shown promising results in providing rich context information including an objective assessment of physical activity level and information on the emotional and physiological state of the person.[15] [25]

Current health coaching systems integrate artificial intelligence (AI)-based conversational agents powered with machine learning and natural language understanding.[23] [31] [32] Such systems are flexible and can respond to users' requests. To enhance users' engagement with health coaching systems, persuasive techniques and gamification design elements can be integrated within the dialog model.[33] [34] This will add a persuasive layer on top of the conversation model. For example, Fadhil and Villafiorita[33] discussed about using a gamification layer within the conversational agent to promote food waste reduction among teenagers.

\section{Text-Based Conversational-Agent-Assisted Coaching}

Most of the approaches in the literature review rely on a software agent to handle all the tasks, and often they cannot accurately handle user needs beyond the technical functionalities.[35] In this research, we go beyond the state of the art, by focusing our approach on a semi-automated system where the dialog flow is controlled by both the conversational agent and a human agent. This research is an extension of our existing PhD research study on health coaching with a human-virtual agent mediated system.

We relied on a task-oriented finite state machine (FSM) architecture to handle the conversational agent dialog. Conversational agent systems are still emotionless, hence a human in the loop can cover this part and provide supports beyond the technical capabilities. The presence of a human in the loop avoids the shortcomings of pure agent-based control, affording an experience in which the system responds appropriately to both verbal and nonverbal cues in dialogues with a user. Self-assessment of how one handles different situations and a feedback about one's performance from a coach enhances learning from the experience.

Rather than relying on SMS services, we used a natural conversation to interact with users, delivered by the conversational agent. A substantial body of evidence spanning several years of research demonstrates that text-messaging interventions have positive effects on health outcomes and behaviors.[11] Most mobile apps and wearable trackers suffer from user abandonment. Instead of a standalone app or wearable tracker, we use a conversational agent inside a messaging app to handle most user interactions and use the wearable tracker as a supportive tool, rather than the main tool to gather user health data.

CoachAI assists the caregiver with initial user profiling and activity assignment. The user profiling is achieved by applying a supervised machine learning approach (namely, support vector machine, SVM[a]) to analyze the collected data and present them to the coach. This is important since users are heterogeneous and have diverse interest and emotional needs. Finally, a mixed human-virtual agent coaching has been found to be more effective than a mere human or agent coaching for health activities.[36]

The platform is domain-independent and general in terms of architecture to integrate it into any health promotion context. The application uses Telegram Bot Platform[b] as the communication channel for user interaction and a web application portal for the caregiver to track and assign user-specific activities. The user is guided by a personalized dialogue with the conversational agent. The dialogue engine is handled by a state machine, where the user switches between states until the dialogue objectives are fulfilled. We apply data analytics to find user-dependent patterns in the link between their health parameters and the activities assigned. In addition, we aim to involve the caregiver only when the user shows no adherence to the plans and no interaction with the conversational agent for a period, defined by the coach.

This research is the first that tests the efficacy of a human-virtual agent mediated conversational agent system for daily lifestyle analysis of users on various perspectives. Our approach helps boosting the efficacy of the caregiver tasks, by automating aspects of their daily tasks through the conversational agent. Given that, our novel approach proves effective, and it could be integrated into various health domains or even outside the health context and reach a vast number of individuals due to its technical scalability. The approach presents innovativeness from different perspectives. This includes the psychology point of view as it examines individual's adherence and provides a summary to the human agent. The approach also provides innovative aspects from a health intervention perspective as it applies a new paradigm to intervention delivery, which includes both the human and the virtual agent. 


\section{Coaching Layer}

The coaching portal refers to the coaching portal and the conversational agent components that interact with each other through other components to form the user-conversational agent interaction and human-conversational agent collaboration. In [Fig. 1], we show the overall system architecture with all the components and actors highlighted. The architecture consists of the coaching layer, the dialog engine, the user clustering component, the conversational agent, and wearable integration.

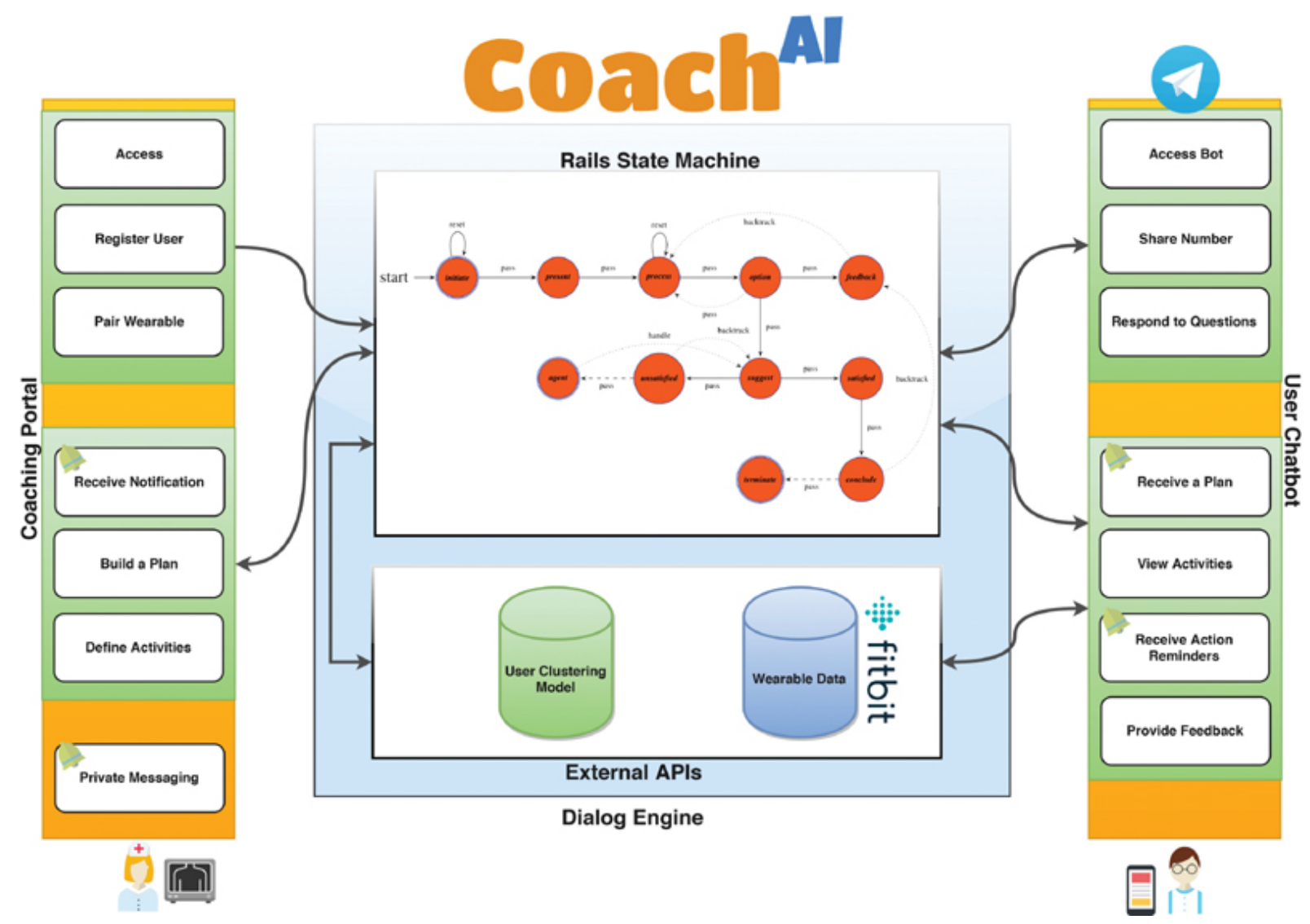

Fig. 1 CoachAI: high-level system architecture.

\section{Coaching Portal}

This is the coaching panel to register and track users ([Fig. 2]). It contains all users' data about their daily exercise and diet, and their overall wellness coping. The coaching portal is a web application dashboard used by the health coach. The dashboard allows the coach to assign initial tasks to the users and check their adherence. One task is formed by several plans, with each plan containing a set of activities. Moreover, the machine-learning model helps the coach to cluster new users into distinct groups. The assigned plans have an associated timeframe, performance validation, and adherence measure. The plan is assigned to the task scheduler to perform the periodic plan assignment and feedback collection. User adherence (high adherent, low adherent) is determined by following a fixed threshold and taking the average of user activity adherence over the plan duration. All the data are aggregated by the conversational agent operating inside the task scheduler. 


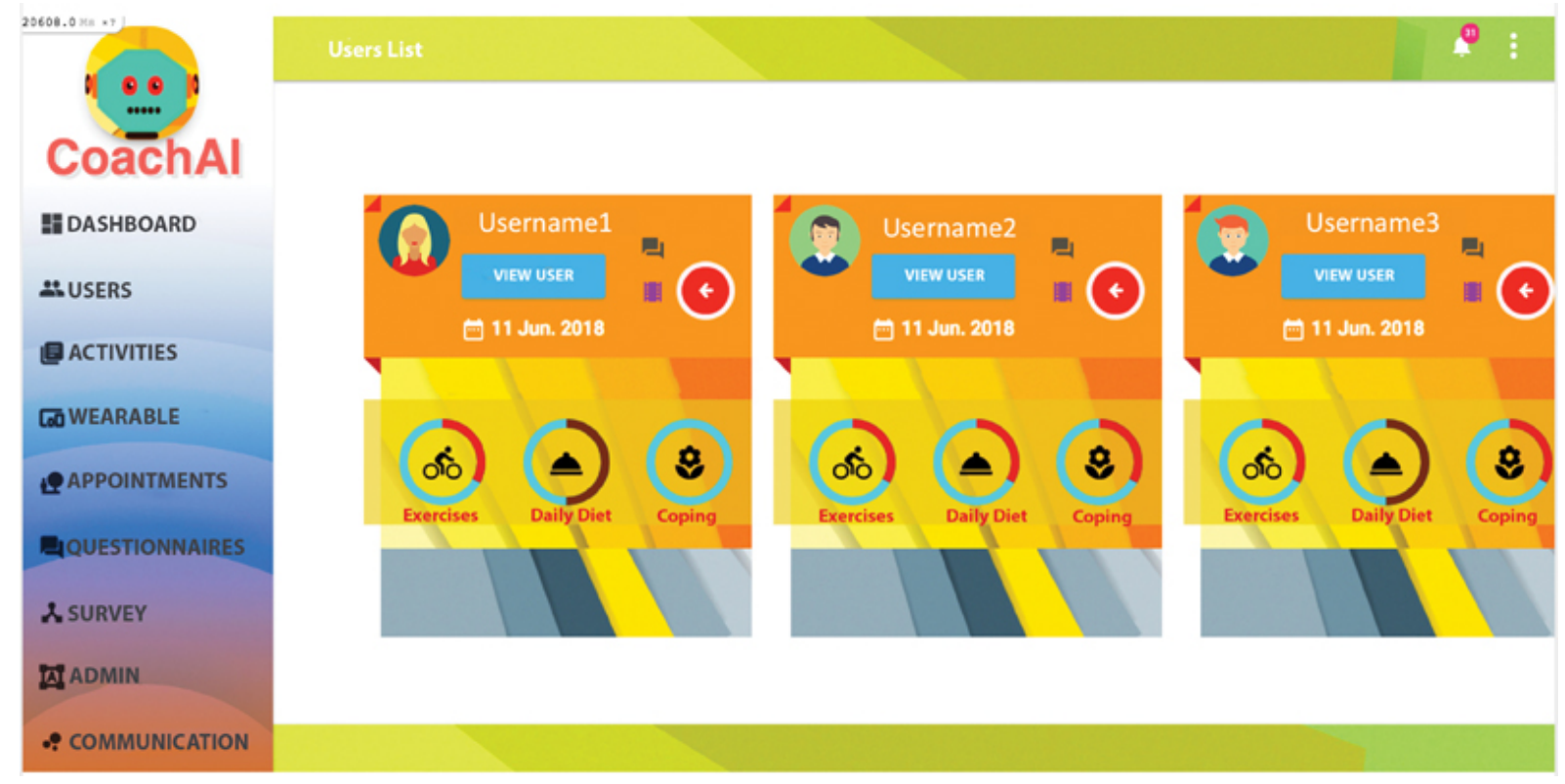

Fig. 2 CoachAI: coaching portal interface.

The human coach can track via the conversational agent protocol various obstacles/problems the user might face, and hence can tune the coaching or treatment accordingly. Existing automated coaching systems are still limited with respect to determining and reacting to user emotional states. A human coach can provide more flexibility to better understand users' preferences and hence tailor the recommendation to their interest. With a human in the loop augmented with the conversational agent, we aim to bring the best of both approaches for the users.

\section{User Conversational Agent}

The concept of conversational agents has already been applied to improve aspects of user lifestyle, through face-to-face conversation, using speech and hand gesture.[37] [38] The conversational agent in CoachAI is based on text conversation and simple graphical elements embedded into the dialog. The conversational agent is the tool users interact with through their mobile application. The user accesses and interacts with the bot on specific topics structurally defined within the hierarchical FSM. The FSM provides a predefined dialog structure to steer the dialog flow. The dialog uses a rule-based model that has predefined conditions to control the flow. The agent guides the user through the states based on users' reply. The initial conversation between the agent and user includes a greeting and some preliminary evaluation chat at the beginning of the interaction. This preliminary evaluation also assesses users' comfort in discussing personal information with the agent. At this step, we gather data about users' BMI, physical activity, diet, stress, and sleep. After this, the agent leads the user through the activity state to provide health promotion activities. The dialog interaction closes with the agent asking the user about their adherence to the activity.

The conversational agent performs two separate phases, namely information gathering and feedback collection. The conversational agent tracks users' adherence and categorizes them into sedentary, mild, or vigorous users. The coach is notified in the case of any user condition deteriorations (see [Fig. 3] for the conversational agent). The conversational agent handles tasks about activities assigned, user feedback per activity, exercises, private messages by the coach, and health intervention questionnaires. 


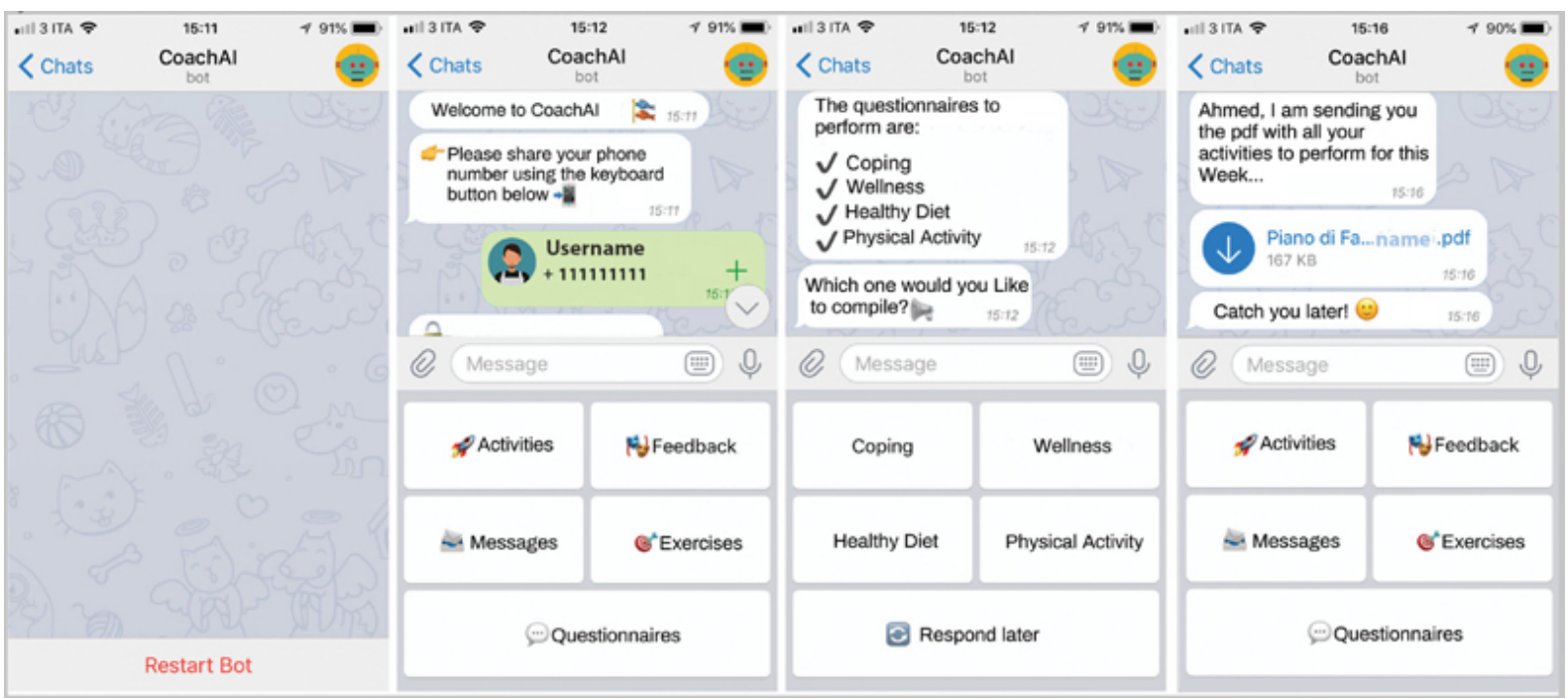

Fig. 3 CoachAI: conversational agent interface.

\section{Task Scheduler}

The scheduler is the mechanic that steers the dialog engine with all the tasks it performs. It handles the state transition when the coach or the user is interacting with the conversational agent. This may include defining a plan with the duration and duties and tracking the plan expiration date and user adherence to the plan. The task scheduler also handles all sorts of notifications between the coaching platform and the conversational agent. The scheduler notifies users when a plan has been assigned and sends them the list with the duration. It provides the appropriate plan and feedback notifications based on the time chosen for the notification. The periodic tasks are handled with a time-based background job scheduler which handles all the periodic events in the system.

\section{Dialog Engine}

The dialog engine is the brain of our conversational agent, which operates on different levels, including user adherence, motivation, and problems. The dialog consists of building the services the platform is supposed to deliver within the conversation. The dialog is based on a structured FSM architecture that offers the capabilities to define the structure for a domain-specific dialog model. Based on the defined pattern, the state machine allows constructing conversation patterns about various topics involved in the conversation. The FSM has unique states that guide the user through a series of steps to achieve their daily goal. The states use the result of previous states and its results are used by the next state. The choice of FSM or automata over the similar approaches was because the number of user tasks is limited. We followed a standard design pattern to structure the most relevant states for health dialog models.[34] For instance, to exercise more or eat healthily, there has to be a finite set of steps a user has to fulfill to reach the end goal of the dialog. Hence, the conversation is a composition of FSM-based dialog that delivers the services through a text-based conversational agent. [Fig. 4] presents the skeleton of the FSM. 


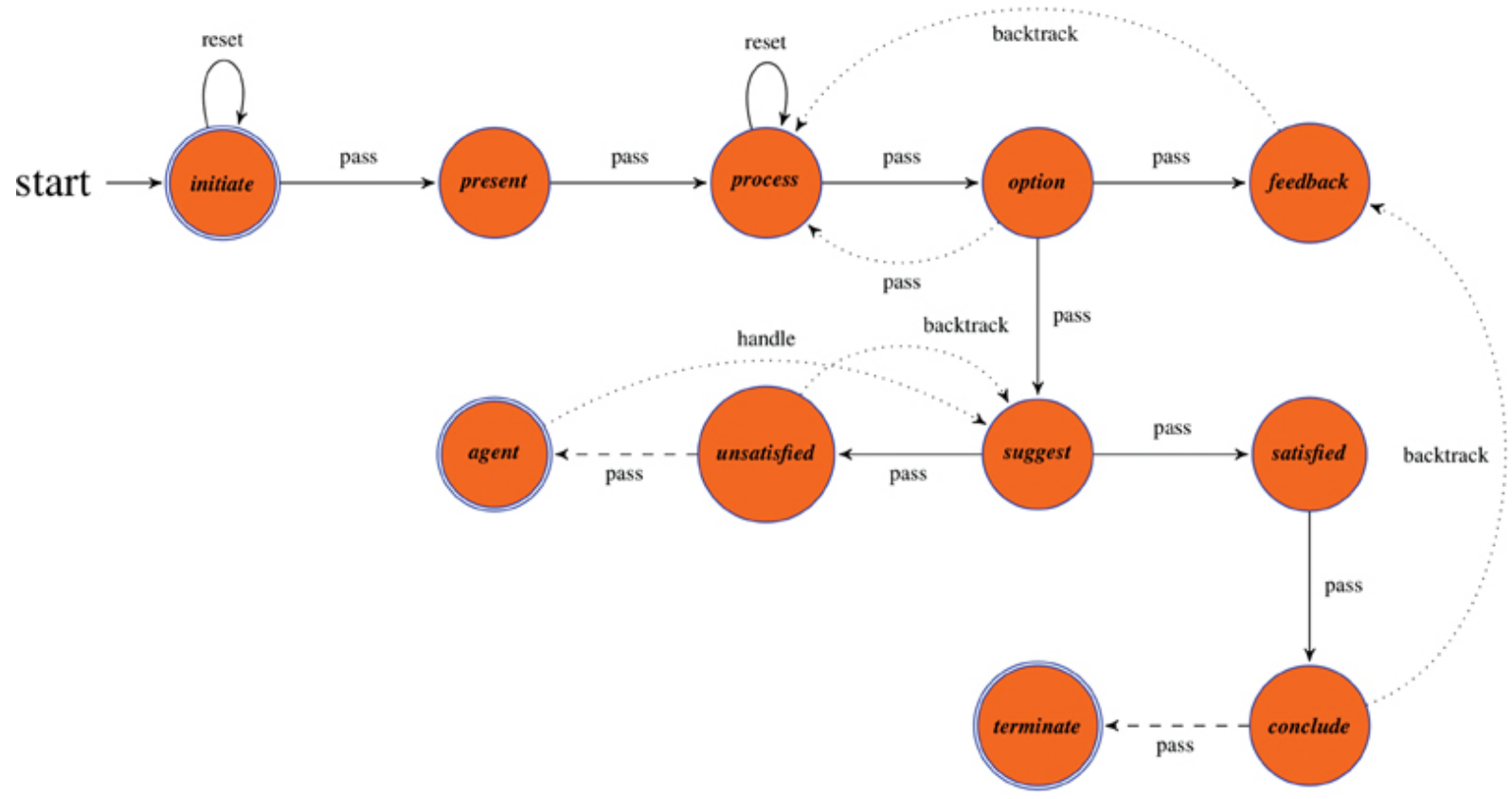

Fig. 4 CoachAI: high-level finite state machine.

The state transition happens when certain conditions are met. When the conversational agent is in the present state, the user is presented with the main functionalities the bot can perform. The user has to select a button or type the functionality they wish to perform to go to options. The option state provides users with information available or asks them to provide their input. Based on user action, the state will take a certain path, i.e., either feedback or suggest state. Unless the state condition is satisfied, the conversational agent will not allow the user to change the state. Once the conditions are satisfied, the user can conclude the dialog and terminate the interaction.

\section{User Classifier}

We used a multiclass SVM classifier to separate new users into three distinct groups based on their initial activity level. The dataset used to train the model was anonymized user data provided by an ambulatory health care clinic. The dataset consisted of real patients' data (age range: 22-74, from both genders) in the form of health record. The data contained information about their demographics, their dietary pattern, their health condition (if any), and their health goal, to mention a few.

Based on the given data, we constructed a health questionnaire and collected data from 375 participants. The overall dataset consisted of 25 features with dependent (e.g., active or inactive individual) and independent variables (e.g., individuals' age, work, daily sedentary time, how much physical activity they perform). The independent variables cause a change in the dependent variables. The features used were mainly about users' physical activity and sedentary lifestyle. We plot each data item as a point in $n$-dimensional space (where $n$ is the number of features) with the value of each feature being the value of a particular coordinate. Then, we perform classification by finding the hyper-plane that differentiates the three classes very well. Based on users' replies to the conversational agent questions, they are categorized into three distinct groups (namely, vigorous, mild, and sedentary users). The model considered some significant features in the data to check for when classifying the users. We used a category $=3$ and a response variable about the physical activity of a person. For a new user, we find the difference with the mean of the clusters and then the closest cluster is chosen. To find the differences we use the lifestyle data and find nearest Euclidean distance from each mean of previous clusters. 


\section{Intervention Delivery}

This approach will be valuable as a decision support system to help the physician in gaining valuable insights on their users. These insights include what motivates/discourages the users, how they feel about a certain activity, their progress with regard to a specific health goal, or what problems they face with their poor lifestyle. The assessment collects data about user dietary habits, physical activity, emotional state, and more. These data help the coach build a plan and assign it to the user. The coach can also deliver standard or customized interventions through the platform. The intervention may consist of some follow-up dialogs with the user or a structured questionnaire for the user to perform to evaluate their condition. The questionnaire consists of a set of questions with their answers and a score for each answer. This approach is based on the Kaiser pyramid model of care.[39] The user answers are scored, and a total score is given for each user's responses. The platform can deliver any type of health and wellness interventions, such as cognitive behavioral therapy.[40]

\section{Methods}

\section{Measurements}

The study investigated the design and interaction aspects, listed below, with the participants' interaction. We used three models throughout the intervention to validate the dimensions listed below. The study used the health action process approach (HAPA)[41] to validate the health behavior engagement. HAPA is a two-layer model: the continuum version is good for analyses and predictions; the stage version is good for interventions. The technology acceptance model (TAM)[42] was used to model how participants come to accept and use the system. Finally, the AttrakDiff model[43] was used to evaluate how participants rate the usability and the design of CoachAI.

During the user conversation with the agent, we capture whether participants self-disclosed personal information to the agent at the baseline evaluation. These self-disclosures included questions like: "How often do you perform physical activity per week?" or "How often did you feel stressed in the past 30 days?" These questions were delivered by the human agent, through the conversational agent. Participants were always given the option to not answer the questions. The study also captured participants' opinions on the healthy diet, physical activity and stress-coping exercises, and whether they felt that these exercises would help them in their lifestyle promotion. Finally, these questionnaires assessed their level of trust in the agent and general satisfaction with the application.

\section{Study Design}

The study initially gathered basic demographic information from participants through a text conversation with the conversational agent. The information contained participants' personal data and personality test about their daily exercise, dietary pattern, and stress. The intervention study lasted for 1 month and participants interacted with the conversational agent daily.

Participants' behavior was measured during the 4-week validation study. For example, participants' physical activity adherence was measured by relying on their conversation with the conversational agent. Self-report measures assessed participants' self-efficacy for (un)healthy eating and active or passive exercise. Participants provided self-reported replies about their exercise measure. The study contains two conditions, baseline (week 1) and intervention (weeks 2, 3, and 4). In the first week, participants were asked to chat with the bot and provide their baseline physical activity and healthy diet intention, together with their stress evaluation. The initial questionnaire and interaction with the conversational agent provided an estimated data about each individual's exercise, diet, and stress level. These data were considered when providing the individual plan per participant and later measured with the intervention condition (the rest of the weeks). The study focused on qualitative analysis to measure participants' behavior change between the baseline and intervention condition. The overall user experience with CoachAI was measured with the validation questionnaires (TAM or AttrakDiff) which was targeting RQ1, whereas their experience changes when they used CoachAI which was measured over their feedback provided to the conversational agent (3 weeks) which also targeted RQ2 and RQ3, and their adherence to the weekly plans was used to measure the RQ3 (HAPA). All participants' data were collected by the conversational agent and anonymized for the duration of the study purpose. Participants' adherence was tracked by the conversational agent and monitored on the coaching portal. 
The emphasis of this study was on participants' interaction with CoachAI and trying to follow the instructions asked by the conversational agent. Moreover, participants received at least a questionnaire with the conversational agent to perform about their overall experience. There were also private messages communicated to the participants at least once a week to report their adherence and provide them with motivational messages. These questionnaires and motivational messages were designed by the caregiver and delivered by one of the researchers through the coaching portal. The motivational messages consisted of positive reinforcement and a feedback on user's overall adherence for the week (e.g., Hi Dave, I realized your stress coping was high, remember to surround yourself with positive people, this is important because...). The messages were fixed and tailored to each participant based on their adherence to the physical activity, healthy diet, and stress management activities. The assigned plans were all validated and structured. All the activities assigned were based on the ChooseMyPlate[44] general recommendations and guidelines and structured by a caregiver.

\title{
Eligibility Criteria
}

The eligibility criteria are a set of predefined characteristics to identify subjects who will be included in the study. To respond to the study objectives and optimize the external and internal validity of the study, we tried to ensure homogeneity of the sample population and increase the likelihood of finding a true association between intervention and outcomes. Therefore, the inclusion criteria selected individuals with sorting in common with each other. For example, the participants were all individuals with sedentary lifestyle and had a poor dietary habit. However, the participants were motivated to pursue a healthy lifestyle. This was confirmed via the initial participants' conversation with the conversational agent and their replies to various motivational messages about physical activity. Most participants were university students or have just obtained their degree.

\section{Participants}

The study involved 22 participants randomly recruited from online social networks and selected based on the criteria given above. At the beginning three participants decided to drop out for personal reasons. The total number of participants was 19 $($ male $=11$, female $=8$, aged: $19-53$, std deviation $=9.371$, range $=34.00$, mean $=28.53$; see $[$ Table 1] $)$, and they were asked to provide their phone number and age for the registration. All subjects were healthy, with no health condition and were motivated to participate in the experiment and perform health promotion activities. Participants were either native Italian speakers or spoke Italian fluently. This was important due to the language-based nature of the study. A reimbursement of $€ 5$ was given to all participants in the form of Amazon coupon. All participants interacted with the bot and performed the initial user profiling.

Table 1

\section{Outcome measure effectiveness criteria}

\begin{abstract}
Effectiveness Criteria
\begin{tabular}{l|l} 
Highly effective & The intervention led to statistically significant improvement on the majority of outcome
\end{tabular} (HE) measures.
\end{abstract}

The intervention was more effective or as effective as comparison groups.

The intervention was more effective than control groups without an intervention or waiting lists.

Low effective (LE) The intervention led to statistically significant improvement on the minority of outcome measures.

The intervention was as effective or less effective than comparison groups. 
Table 1

\section{Outcome measure effectiveness criteria}

\begin{tabular}{|l|l|}
\hline Effectiveness & Criteria \\
\hline
\end{tabular}

The intervention was more effective than control groups without an intervention or waiting lists.

\begin{tabular}{|l|l} 
Ineffective (I) The intervention led to no statistically significant improvements on any of the outcome
\end{tabular} measures.

The intervention was no more effective than control groups without an intervention or waiting lists.

\section{Procedures}

The procedure starts with the human agent registering all the users, then the conversational agent asks them to perform the initial questionnaire to evaluate aspects of their diet, physical activity, and other health parameters (baseline). All participants installed the Telegram application and started using the conversational agent at the same time, using their own mobile devices. On concluding the initial questionnaire, the human agent receives a notification indicating user-profile completion. The human coach then can either define a new health plan adherent with user condition or assign a prebuilt plan from the activity pool. All the plans have a category, a description, a periodic event triggering time, and an expiration time. Upon plan submission, the conversational agent notifies the users about the plan availability, then collects their feedback. The feedbacks are represented by users' replies to the conversational agent and how often they interact with the system. Over time, the conversational agent also tracks user's adherence to the plan by measuring the average adherence to the activities defined. For example, in a weekly plan the conversational agent measures the users' average adherence to all the activities. The adherence is collected in two ways, either by providing answers to the conversational-agent questions or aggregating the data from user wearable, in case paired with CoachAI. Due to the experiment duration and lack of wearable availability, we did not test the data collection through the wearable and restricted it to the conversational agent. [Fig. 5] below shows the experiment setup we followed throughout the CoachAI experiment design process.

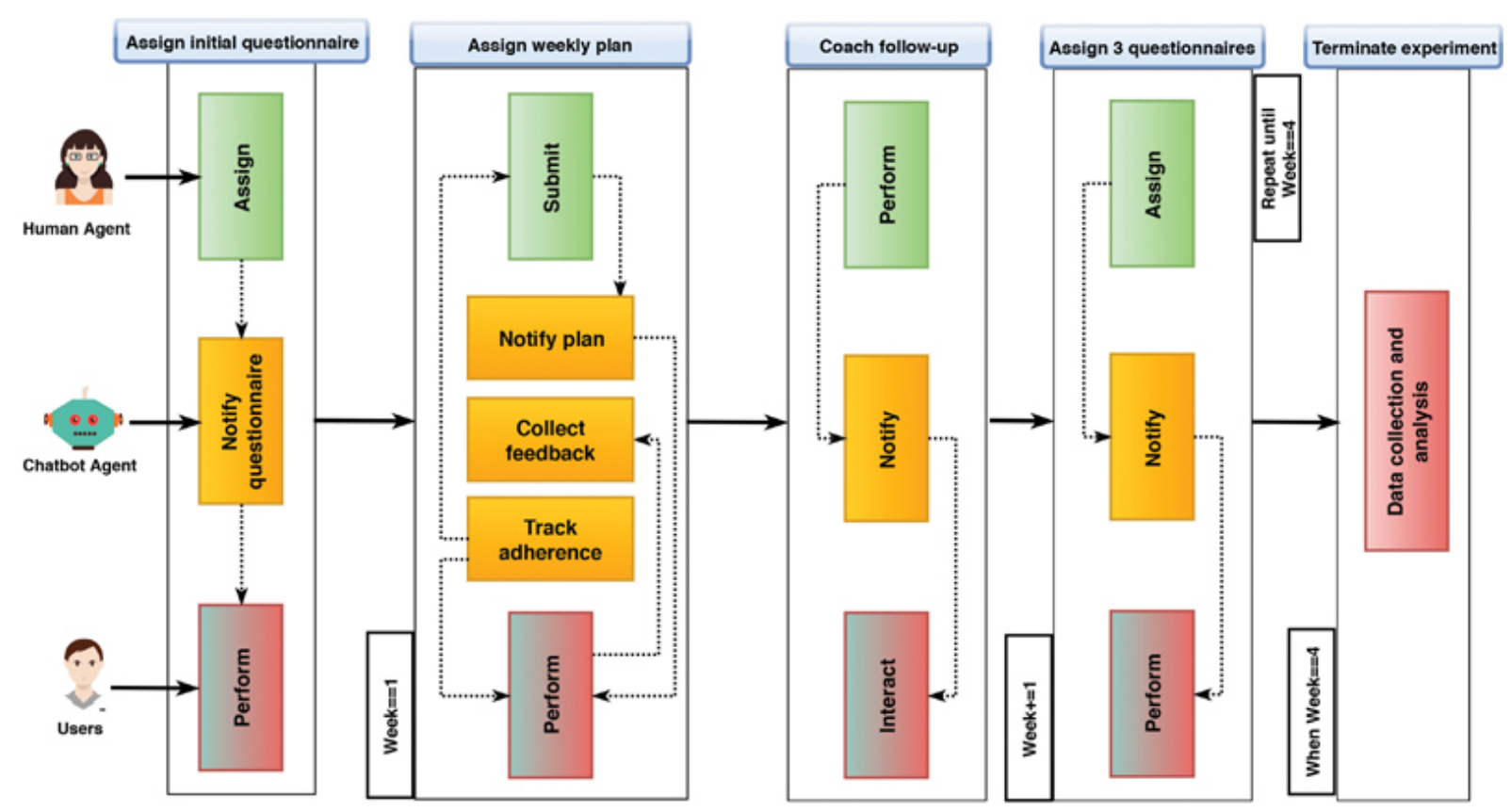

Fig. 5 CoachAI experiment design process. 
In this pilot study we focused on healthy diet, exercise, emotion recognition, and mindfulness tips provided to users in the form of structured activities. The underlying concept was to have participants go through the process of health coaching that is personalized and assigned by a human agent.

At the end of each week the coach performed a follow-up with the users through the private messaging channel. The followup consists of a message sent to the users which contains motivational reinforcement and a feedback on their overall compliance to the plans. The messages are all prebuilt on the web dashboard and tailored to each user. These messages are communicated to participants' Telegram application by the health coach. Moreover, three questionnaires (namely, the TAM, HAPA, and AttrakDiff) are delivered to the users over the conversational agent at the end of every week. These questionnaires measure aspects of user's experience, expectations, and other interaction-related parameters with CoachAI. This procedure lasted for 4 weeks and was terminated at the end of the fourth week.

\section{Evaluation Objectives}

Our objective was to measure participants' engagement in health behavior change activities, their acceptance and use of the system, and their rating of system usability and design. Therefore, three methods (namely, HAPA, TAM, and AttrakDiff) were used to validate our evaluation objectives and measure any outcome that might emerge from these validations. The intervention-measured effectiveness was categorized based on the studies of Lentferink et al[45] and Morrison et al.[46] This divides the intervention outcome into three catagories, namely highly effective, low effective, and ineffective interventions (see [Table 1] for the effectiveness criteria). We listed the outcome measure per objective based on the results we obtained from users' experiment. In [Table 2] we list the methods and the evaluation objectives with the outcome per method.

\begin{tabular}{|l|l|l|}
\hline \multicolumn{1}{|c|}{ Methods and evaluation objectives } \\
\hline Evaluation objectives & Methods & Outcome measure \\
\hline Adherence analysis & TAM & HE \\
\hline Attitude and intention & TAM & HE \\
\hline Pragmatic, hedonic, appealing, social qualities & AttrakDiff model & HE \\
\hline Motivation and volition phase & HAPA & I \\
\hline Familiarity analysis & AttrakDiff model, TAM & HE, HE \\
\hline
\end{tabular}

Abbreviations: HE, highly effective; I, ineffective; LE, low effective; HAPA, Health action process approach; TAM, Technology acceptance model. 


\section{Results}

We analyzed the weekly data collected using the three models to evaluate users' overall experience with CoachAI. This evaluation included user's adherence to the conversational agent activities, their attitude and intention to use the system, the pragmatic, hedonic, appealing, and social qualities emerged from their interaction, and their familiarity analysis. We also analyzed user's topic-specific interaction preferences and their opinion about the conversational agent.

\section{Adherence Analysis}

The system calculated participants' overall adherence to the plan and reported their total adherence at the end of each plan expiration. The adherence was categorized into high and low adherence groups. Users performing above the threshold were categorized as highly adherent, and vice versa. Participants were categorized according to their adherence to the plan into high and low adherence, with respectively 10 and 9 participants each. The TAM dimensions were analyzed with a multivariate ANOVA (MANOVA) with "adherence level" as between subject factor. The MANOVA shows a significant effect of the between subject factor for usefulness $(F(1,16)=6.5, p<.01)$, fun $(F(1,16)=4.5, p<.01)$, and attitude $(F(1,16)=6.9, p<.01)$. No differences were found for the ease of use and intention. [Fig. 6] shows the result of the user adherence versus the TAM dimensions.

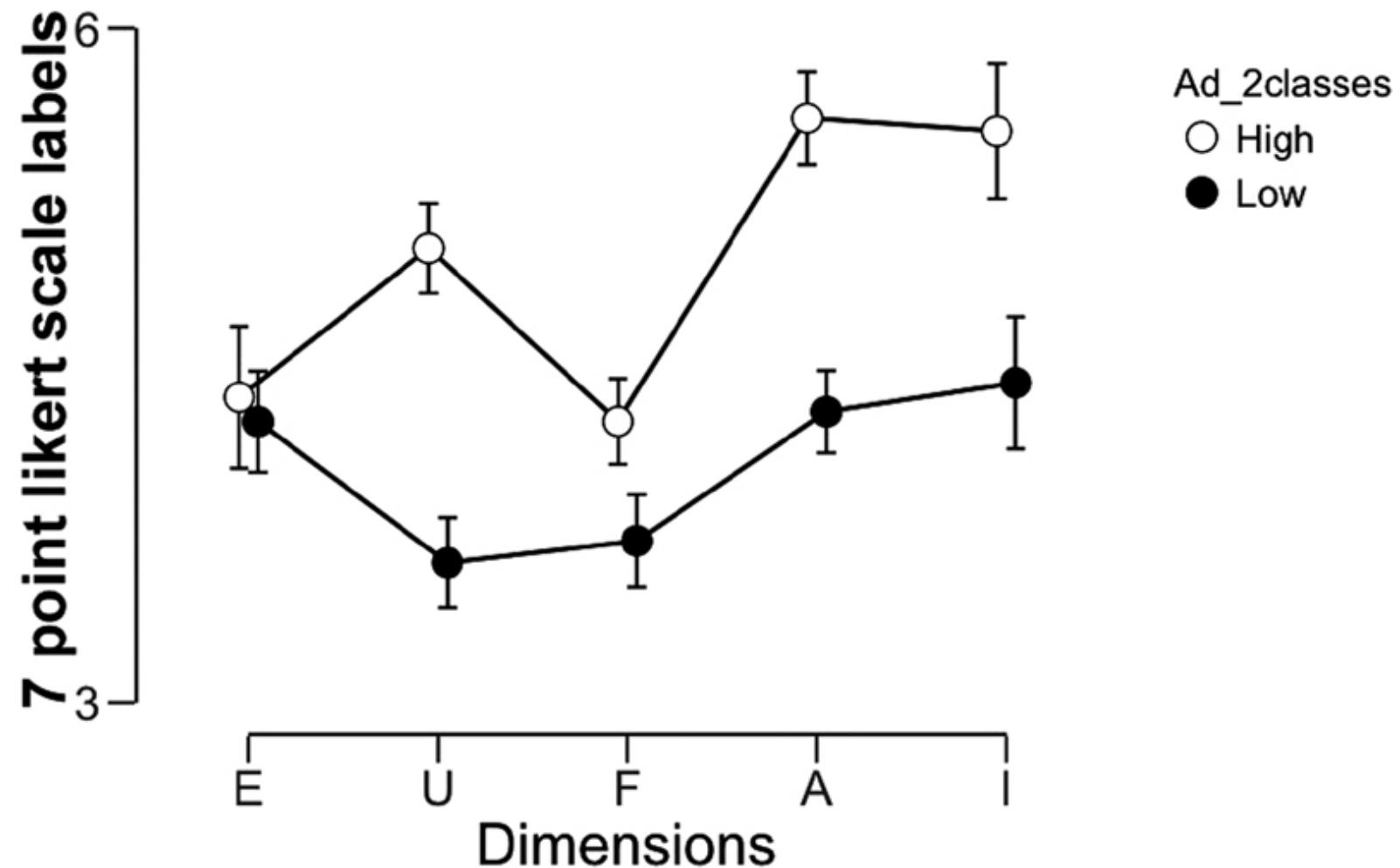

Fig. 6 User adherence analysis and TAM. E (ease of use), U (usefulness), F (fun), A (attitude), I (intention). TAM, technology acceptance model.

\section{Attitude and Intention}

Participants highly rated both attitude and intention toward using the system. Most participants gave a rating above average to both dimensions. Considering the questionnaire on the dimensions of TAM ([Fig. 7]), a one-sample $t$-test was used to compare the means for each scale to the scale middle value (score $=4)$. The scales "ease of use," "attitude," and "intention" are significantly higher than the middle score (respectively: $t(17)=4.9, p<.01 ; t(17)=2.5, p<.05 ; t(17)=3.1, p<.01)$. A repeated measure ANOVA (with time as a within factor) showed a difference only for the scale "intention" between the three different weeks. Post-hoc analyses reveal that the scores for this scale at week 1 were higher than week 2 and week 3 ([Fig. 8]). 
TAM dimensions (average score)

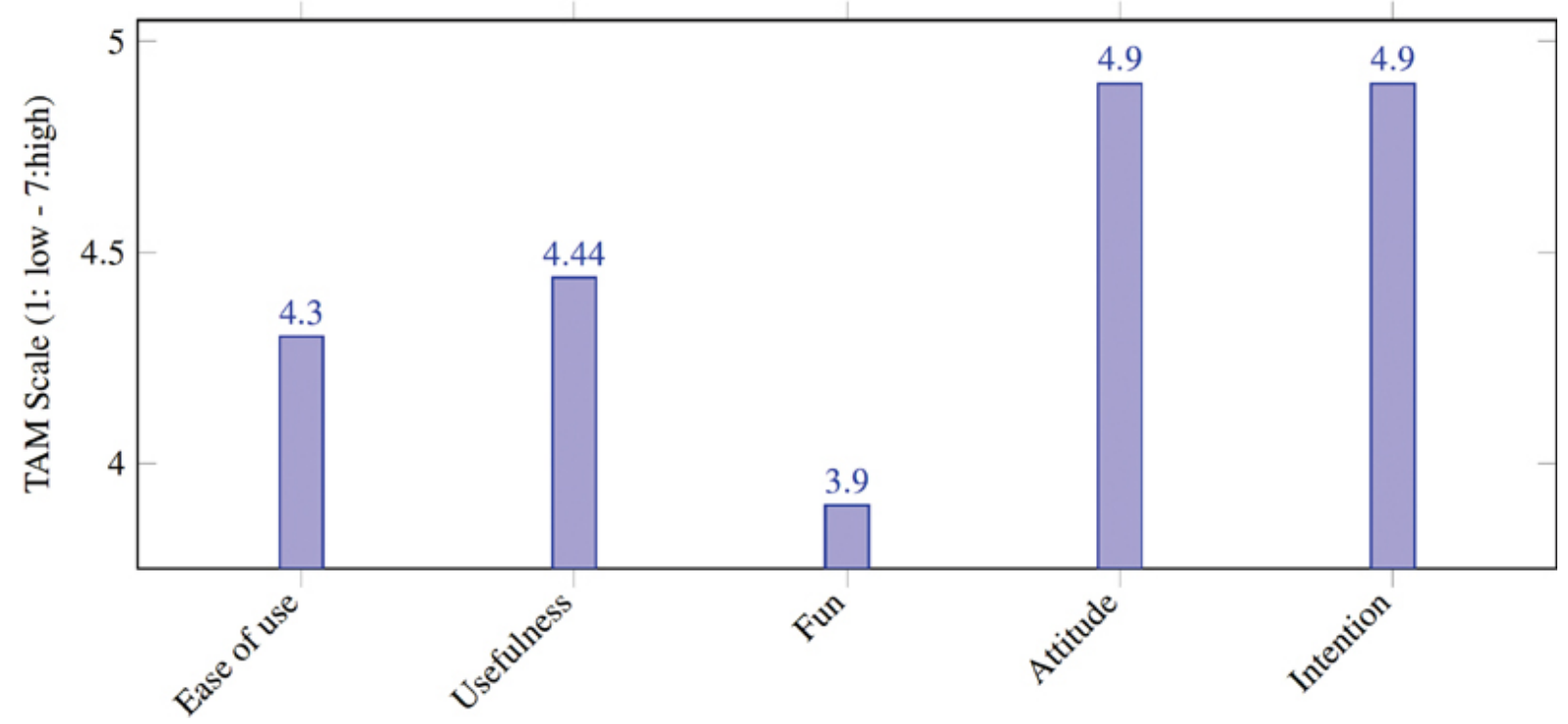

Fig. 7 TAM dimensions (average score). TAM, technology acceptance model.
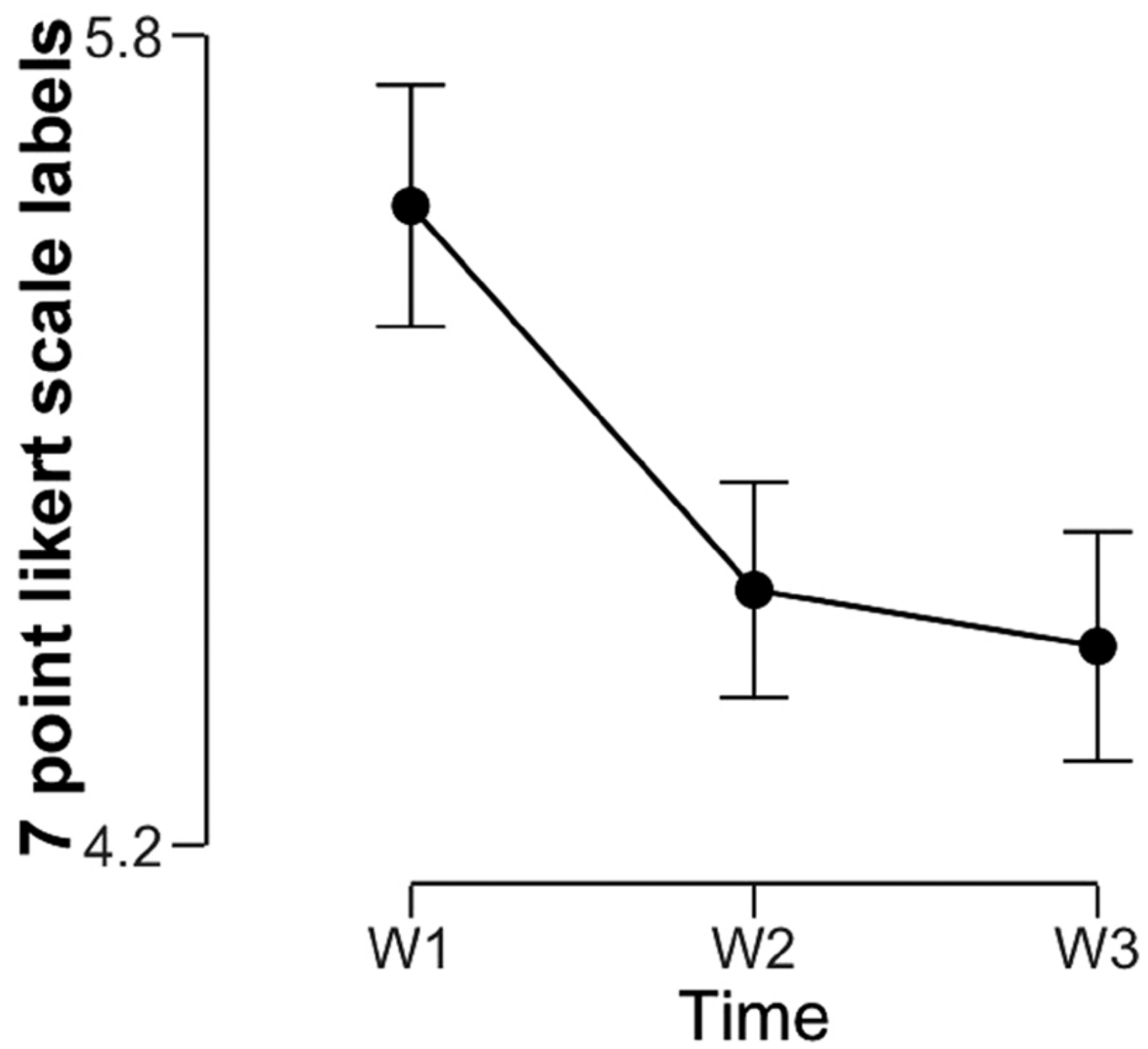

Fig. 8 TAM post-hoc analyses between weeks. TAM, technology acceptance model. 


\section{Pragmatic, Hedonic, Appealing, and Social Qualities}

We analyzed these qualities based on users' responses over 4 weeks. The results of the AttrakDiff questionnaire are summarized in [Fig. 9] below: a one-sample $t$-test was used to compare the mean scores for each dimension to the scale middle value corresponding to "neutral" (score $=4$ ). The test shows that the average scores are statistically higher than 4 for each dimension: pragmatic $(t(17)=5.41, p<.01)$, hedonic $(t(17)=3.4, p<.01)$, appealing $(t(17)=4.2, p<.01)$, and social $(t(17)=2.6, p<.05)$.

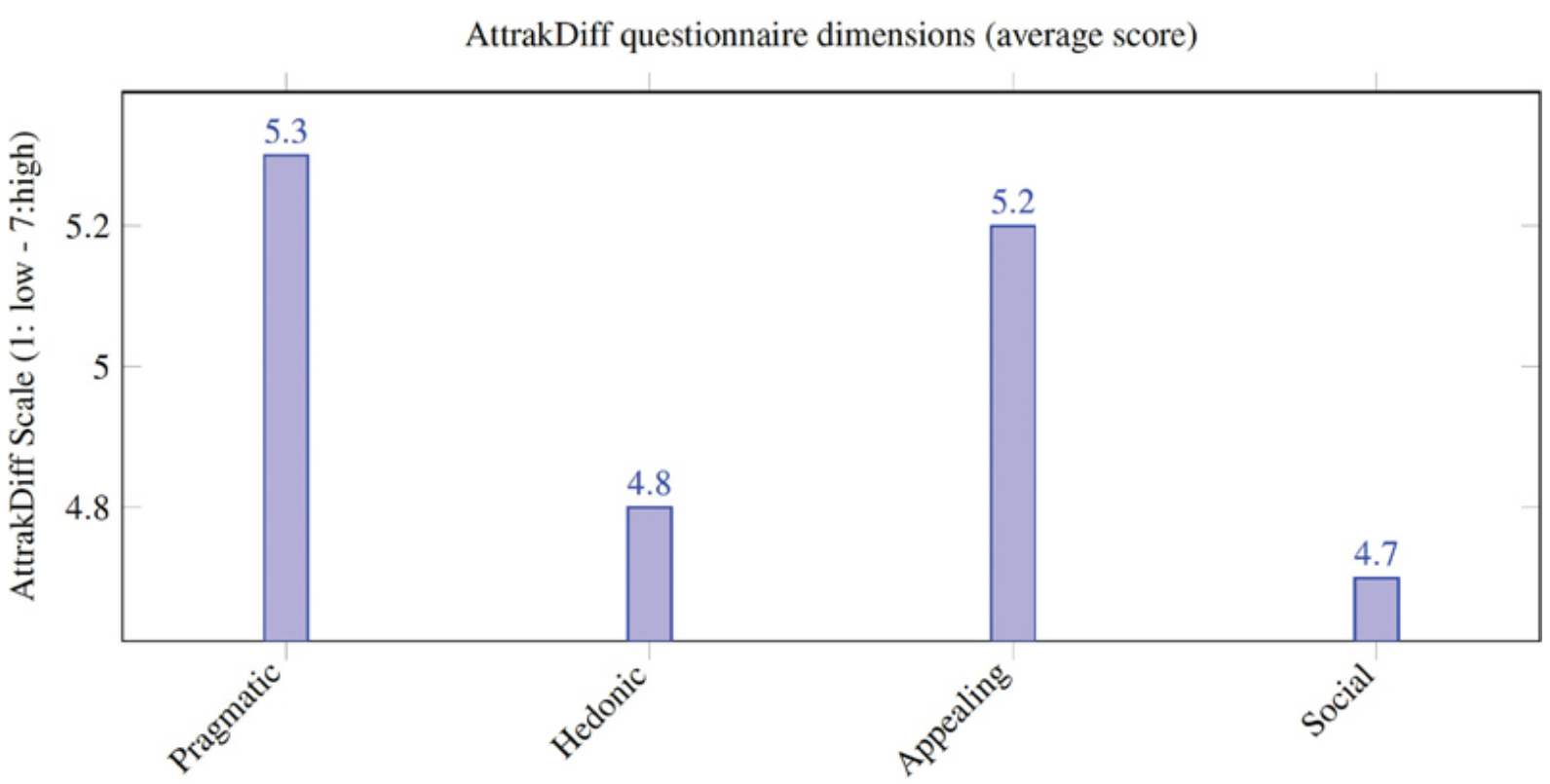

Fig. 9 AttrakDiff questionnaire dimensions (average score).

\section{Motivation and Volition Phases}

Participants' intention to change risky behavior or adopt a new measure was evaluated through the motivation and volition phases of the HAPA model.[41] [47] The HAPA stage model was adopted to measure participants' intention to change or improve self-efficacy, outcome expectancy, and risk perception. Users in this model can either progress their behavioral intention to change or they may also fall into relapses and recycle through the stages. We compared these stages with individual's overall adherence to the activities. Participants were asked to provide their answers to the HAPA questionnaire over the month. We analyzed individual's data about physical activity, healthy diet, and overall health expectation and mapped these data with the perceived self-efficacy, outcome expectancy, and risk perception. A repeated measure ANOVA (with time as a within factor) showed no difference between the three weeks; the scores remained unchanged for the physical activity, healthy diet, and mental wellness intention.

Users' health expectancy data were collected with questionnaires at three points through the conversational agent. We mapped individuals' health expectancy data with their adherence to measure their risk perception, positive outcome expectancies, action self-efficacy, and behavioral intentions. The analysis revealed no statistically significant results when analyzed within subject and between subject effects. [Fig. 10] shows the comparison between individuals' physical activity intention, healthy diet intention, mental wellness, health expectancy, and overall adherence. 

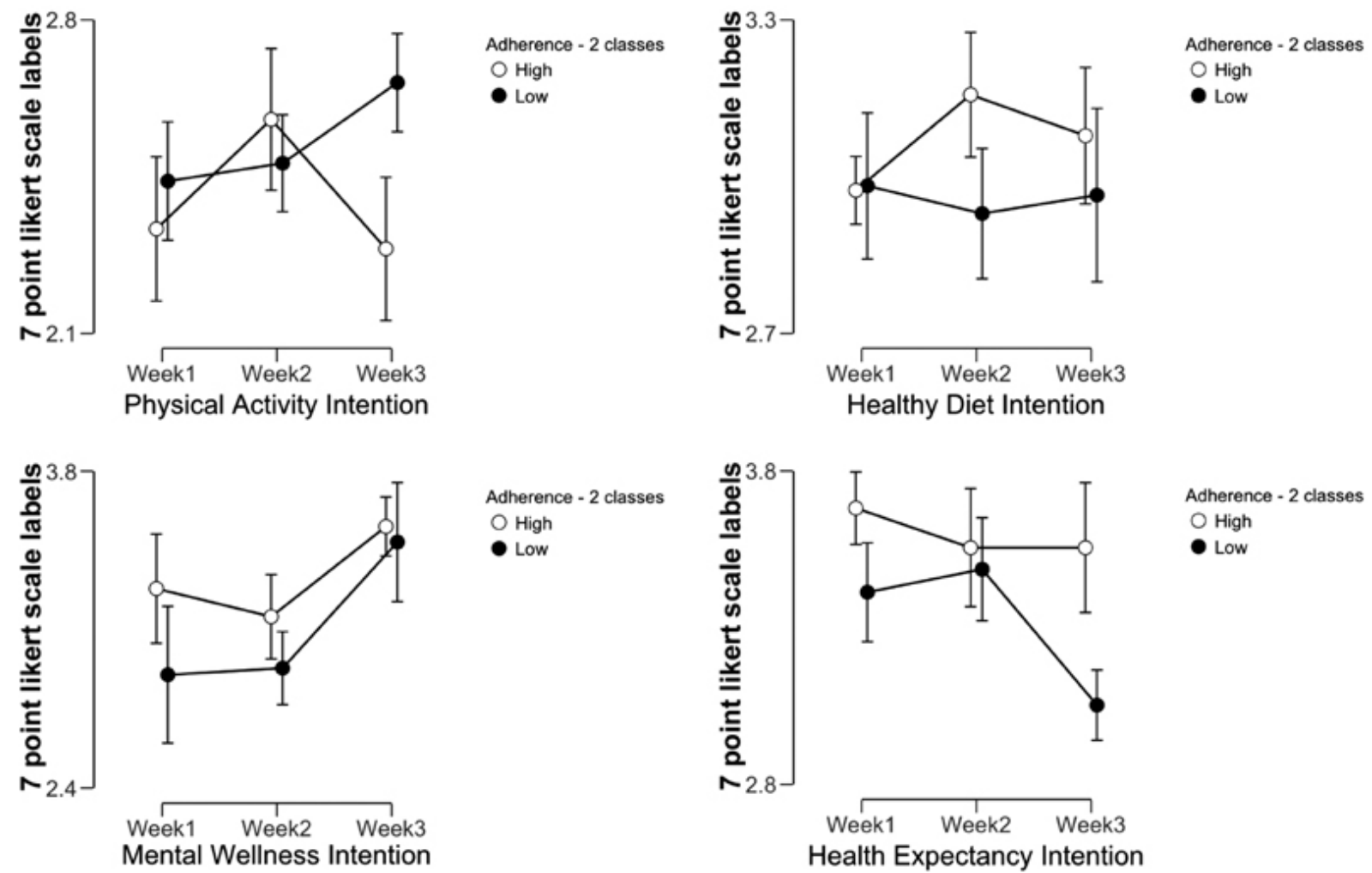

Fig. 10 Physical activity, healthy diet, mental wellness, and health expectation versus individual's adherence.

The high and low variables refer to individual's overall adherence to the plans over the 3 weeks. We measured these two variables by calculating the average of user's weekly activities and dividing them by the total number of activities assigned. We compared these two variables against users' responses to each of the physical activity, healthy diet, mental wellness, and health expectancy measures collected by the HAPA questionnaires.

\section{Conversational Agent Familiarity Analysis}

We ran a multivariate ANOVA test to measure this dimension against the AttrakDiff and TAM dimensions. Based on selfrated scale, participants were categorized by their familiarity with conversational agents and the frequency of use of such technology. Participants were grouped in two categories: high-familiarity (11 participants) and low-familiarity (8 participants). A multivariate ANOVA was used to test for differences between these two categories with AttrakDiff and TAM dimensions. Regarding AttrakDiff, the analysis shows a significant effect of the between factor for the pragmatic $(F(1,16)=4.9, p<.01)$, hedonic $(F(1,16)=7.8, p<.01)$, and appealing $(F(1,16)=4.8, p<.05)$ dimensions. Participants more familiar with conversational agent applications scored higher in all the dimensions. Considering the TAM dimensions, the analysis shows an effect for the usefulness $(F(1,16)=6.2, p<.05)$ and fun $(F(1,16)=8.4, p<.05)$ dimensions. Participants with high familiarity with the conversational agent technology reported higher scores in these categories (see [Fig. 11]).
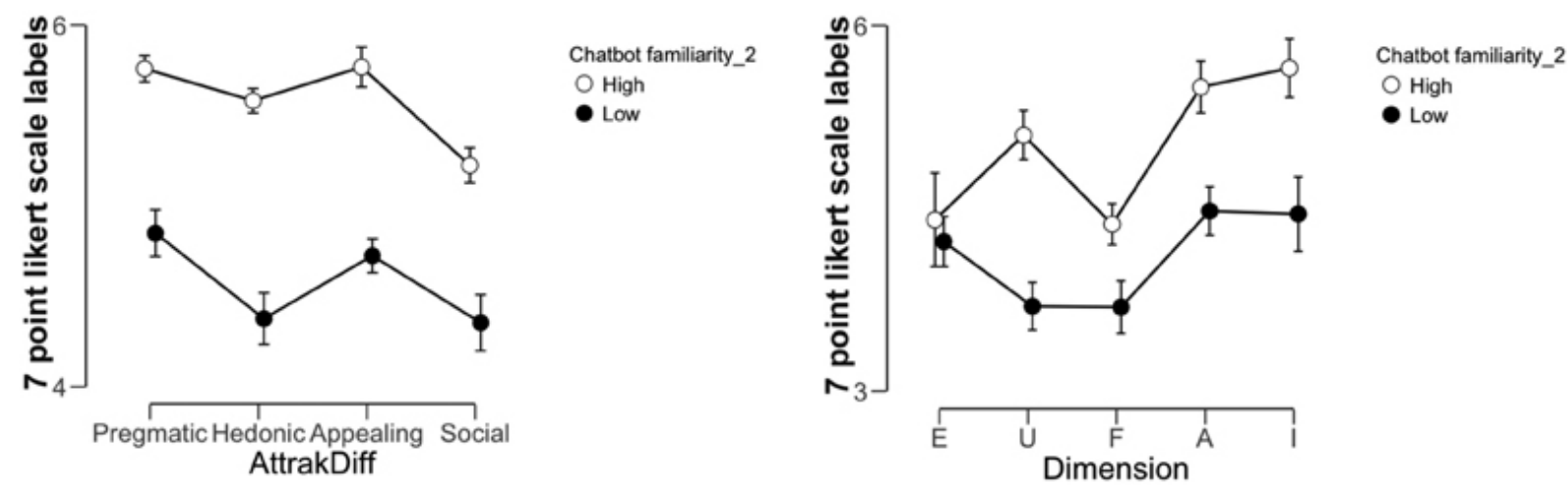

Fig. 11 Conversational agent familiarity dimension versus AttrakDiff and TAM dimensions. TAM, technology acceptance model. 


\section{Topic-Specific Interaction Preferences}

We performed a self-evaluation via the conversational agent at the end of user's feedback to their activities to validate their interaction with the conversational agent and measured their experience with the system when performed a topic-specific activity. The evaluation was performed at the end of each week. For example, if the users performed a physical activity plan, they were asked to indicate their preferences to be coached by a human agent, a virtual agent, or a combination of the two. A fixed set of validation questionnaires were asked to the participants through the conversational-agent channel.

Interestingly, participants following a physical activity plan indicated their preferences to a virtual coach to track their physical activities. Whereas, those following a healthy-diet plan preferred having a human agent to provide them with daily dietary activities and monitor their progress. Finally, participants following a mental wellness activity chose a combination of a human agent and a virtual agent to monitor and assist their anxiety and stress coping (see [Fig. 12] below for the differences). We believe this result is due to the nature of each topic, which may defer in terms of needs and user expectations. In addition, some topics are more emotional bound than others and vary in the level of health complexity. For example, users in the mental-wellness group may need support beyond the technological capabilities, hence having a human in the loop will provide them with the emotional support and can change the communication tone which is crucial in such domains.[12]

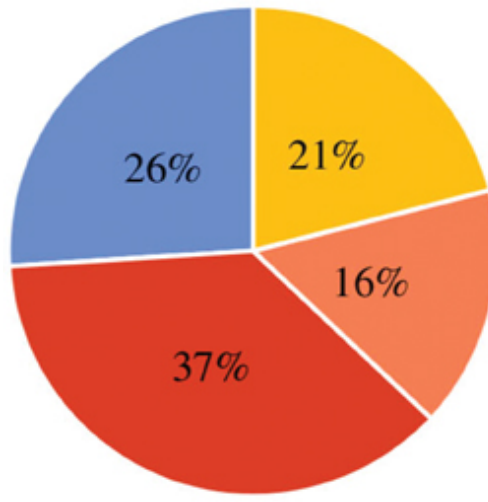

Physical activity coaching style

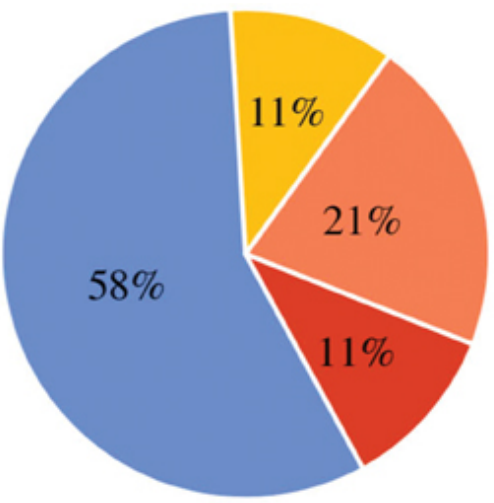

Healthy diet coaching style

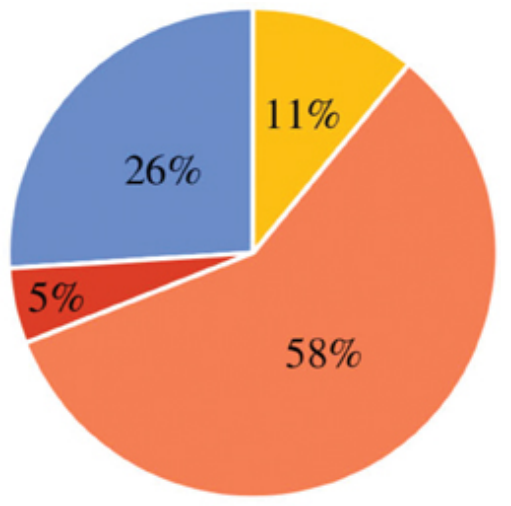

Mental wellness coaching style $\square \quad$ A human agent

$\square$ A chatbot agent $\square \quad$ A combination of the two

$\square \quad$ Unknown

Fig. 12 Participants - health coaching preferences by topic. Left (physical activity group), middle (healthy diet group), and right (mental wellness group).

\section{Users' Opinion About the System}

We gathered system-specific data from users through the conversational agent, using a 7 Likert scale. The preliminary results showed that the participants were highly satisfied with the agent, had high trust in the agent, and were willing to reveal sensitive personal information to the agent (e.g., their overall stress level). Hence, our result indicates that providing a conversational agent support to individuals with poor lifestyle is feasible; however, whether using a virtual agent, a human agent or a combination depends on the context of health domain. 


\section{Discussion and Limitations}

A text-messaging-based mobile application is a powerful tool for behavioral change interventions, due to its widely availability, inexpensive, and ease of use.[21] [48] A substantial body of evidence spanning several years of research demonstrates that text-messaging interventions have positive effects on health outcomes and behaviors.[11] [21] CoachAI is a messaging-based conversational agent built to support the development, classification, and delivery of both individual- and group-based health interventions. The health activities were sent recurrently (e.g., daily, weekly, on weekends, or weekdays) or at a scheduled date and time decided by the coach. Across the experiment there was strong support for the research questions. Overall, the experimental results clearly led to a positive answer to RQ1. There was some noticeable user experience emerged from using CoachAI. For example, participants positively evaluated having a human coach in the background to fine-tune their activities and message them over the conversational agent.

Users' experience changed and varied while performing their activities, which confirmed our RQ2. For example, while some participants showed constant interaction frequency with the conversational agent, others had an increasing or decreasing interaction frequency based on their timestamp or day of the week.

Finally, there was a strong support for RQ3; individuals performing different health activities revealed their preferences to a direct human agent support, or the conversational agent support, or a combination of the two. For example, based on participants' interaction domain and the mechanisms used to deliver the activities, they had various opinions about the coaching style used.

The research concluded that building the technology in a user familiar environment is an effective way to increase factors of technology sustainability. The users positively perceived the activity sessions when they are planned by a human coach. Hence, in some domains the support of a human agent is clearly more motivating and engaging with respect to the virtual trainer that reveals itself alone insufficient to fulfill users' needs. Our 1-month pilot has demonstrated that individuals have a generally positive reaction to a virtual agent providing them with health interventions and tracking their feedback.

Participants felt the agent was interesting, easy to talk to, and expressed high levels of trust in the agent and desire to work with the conversational agent again. Most participants felt that their conversation with the agent would help them in their health promotion and most were willing to reveal personal information about their daily life to the conversational agent.

That said, several limitations worth mentioning emerged from the intervention study. First, although the activities were clinically validated and clearly guided, they were assigned by a researcher and not a health care provider. Moreover, participants were randomly chosen, healthy subjects and not involved in the testing as users. This was because of the study focus, which was on validating participants' experience with the conversational agent. Second, a long-term follow-up is required with health professionals and real users to clinically validate the efficacy of the platform in assisting caregivers and supporting their users. We acknowledge these as a potential limitation to the generalization of the results.

\section{Conclusion and Future Work}

Existing health applications for lifestyle promotion mostly focus on handling users' condition and recommending activities and plans to improve it. We took a different approach and focused first on increasing caregivers' time efficiency and decreasing their fatigue during user follow-up, and hence improving the efficiency of care. Health coaching systems with conversational agent are novel and can offer a lot of benefit to the mHealth domain both for health care providers and users. Conversational agents cannot replace what humans are good at, but they can provide an interesting channel to support users in delivering services through a simple conversation-delivering personalized care.

Our system was evaluated in a 1-month validation study, where participants interacted with the conversational agent on different health-related topics provided with the health coaching portal and providing them with clinically validated health activities. The study provided a set of dimensions when building conversational-agent-powered health-intervention tools. The results validated some of the questions and provided interesting insights when using conversational agents in health coaching systems. Future work will try to overcome some of the limitations emerged during the experiment. Finally, a randomized control trial experiment with/without a human coach (with control and intervention group) will validate if users perform more activities when supervised by a human agent over the conversational agent. 


\section{References}

1 Epstein LH, Gordy CC, Raynor HA, Beddome M, Kilanowski CK, Paluch R. Increasing fruit and vegetable intake and decreasing fat and sugar intake in families at risk for childhood obesity. Obes Res 2001; 9 (03) 171-178

2 Bech P, Olsen LR, Kjoller M, Rasmussen NK. Measuring well-being rather than the absence of distress symptoms: a comparison of the SF-36 Mental Health subscale and the WHO-Five Well-Being Scale. Int J Methods Psychiatr Res $2003 ; 12$ (02) 85-91

3 Smith KA, Dennis M, Masthoff J. Personalizing reminders to personality for melanoma self-checking. Paper presented at: Proceedings of the 2016 Conference on User Modeling Adaptation and Personalization; July 13, 2016; New York, NY: ACM; 85-93

4 Sawyer MG, Arney FM, Baghurst PA., et al. The mental health of young people in Australia: key findings from the child and adolescent component of the national survey of mental health and well-being. Aust N Z J Psychiatry 2001; 35 (06) 806814

5 Dol A, Kulyk O, Velthuijsen H, van Gemert-Pijnen JE, Strien TV. The use of dialectical dialogues in a personalized virtual coach for obese emotional eaters: A research protocol for a field study among target group. Paper presented at: eTELEMED 2017: The Ninth International Conference on eHealth, Telemedicine, and Social Medicine; Welmington, DE: International Academy, Research, and Industry Association (IARIA), 2017

6 Turner-McGrievy GM, Beets MW, Moore JB, Kaczynski AT, Barr-Anderson DJ, Tate DF. Comparison of traditional versus mobile app self-monitoring of physical activity and dietary intake among overweight adults participating in an mHealth weight loss program. J Am Med Inform Assoc 2013; 20 (03) 513-518

7 Schoffman DE, Turner-McGrievy G, Jones SJ, Wilcox S. Mobile apps for pediatric obesity prevention and treatment, healthy eating, and physical activity promotion: just fun and games?. Transl Behav Med 2013; 3 (03) 320-325

8 Bosomworth D. Mobile marketing statistics 2015 . Leeds: Smart Insights (Marketing Intelligence) Ltd. 2015

9 Seiler R, Hüttermann M. E-Health, fitness trackers and wearables-Use among Swiss students. Paper presented at: Advances in Business-Related Scientific Research Conference 2015 Proceedings; Venice, Italy. 2015

10 Clawson J, Pater JA, Miller AD, Mynatt ED, Mamykina L. No longer wearing: investigating the abandonment of personal health-tracking technologies on craigslist. Paper presented at: Proceedings of the 2015 ACM International Joint Conference on Pervasive and Ubiquitous Computing; September 7, 2015; New York, NY: ACM; 647-658

11 Hall AK, Cole-Lewis H, Bernhardt JM. Mobile text messaging for health: a systematic review of reviews. Annu Rev Public Health 2015; 36: 393-415

12 Fadhil A, Matteotti C, Armellin G, Villafiorita A, Betti D. Coach Me: a platform for promoting healthy lifestyle. Paper presented at: Proceedings of the 18th International Conference on Human-Computer Interaction with Mobile Devices and Services Adjunct; September 6, 2016; New York, NY: ACM; 1077-1080

13 Fadhil A, Gabrielli S. Addressing challenges in promoting healthy lifestyles: the al-conversational agent approach. Paper presented at: Proceedings of the 11th EAI International Conference on Pervasive Computing Technologies for Healthcare; May 23, 2017; New York, NY: ACM; 261-265

14 Fadhil A, Schiavo G, Wang Y, Yilma BA. The effect of emojis when interacting with conversational interface assisted health coaching system. Paper presented at: Proceedings of the 12th EAI International Conference on Pervasive Computing Technologies for Healthcare; May 21, 2018; New York, NY: ACM; 378-383

15 Bardus M, Hamadeh G, Hayek B, Al Kherfan R. A self-directed mobile intervention (WaznApp) to promote weight control among employees at a Lebanese university: protocol for a feasibility pilot randomized controlled trial. JMIR Res Protoc 2018; 7 (05) e133

16 Fadhil A. A Conversational Interface to Improve Medication Adherence: Towards AI Support in User`s Treatment (preprint arXiv:1803.09844). Available at: https://arxiv.org/ftp/arxiv/papers/1803/1803.09844.pdf . Accessed April 15, 2019 17 Fitzpatrick KK, Darcy A, Vierhile M. Delivering cognitive behavior therapy to young adults with symptoms of depression and anxiety using a fully automated conversational agent (Woebot): a randomized controlled trial. JMIR Ment Health 2017; 4 (02) e19

18 Brawley LR, Rejeski WJ, King AC. Promoting physical activity for older adults: the challenges for changing behavior. Am J Prev Med 2003; 25 (03) (Suppl. 02) 172-183

19 Abdelrazek M, Ibrahim A, Cain A, Grundy J. Vision: mobile ehealth learning and intervention platform. Paper presented at: Proceedings of the 5th International Conference on Mobile Software Engineering and Systems; May 27, 2018; New York, NY: ACM; 252-256

20 Ibrahim ZM, Fernández de la Cruz L, Stringaris A, Goodman R, Luck M, Dobson RJ. A multi-agent platform for automating the collection of user-provided clinical feedback. Paper presented at: Proceedings of the 2015 International Conference on Autonomous Agents and Multiagent Systems; May 4, 2015; Richland, WA: International Foundation for Autonomous Agents and Multiagent Systems; 831-839

21 Kitsiou S, Thomas M, Marai GE. , et al. Development of an innovative mHealth platform for remote physical activity monitoring and health coaching of cardiac rehabilitation patients. Paper presented at: 2017 IEEE EMBS International Conference on Biomedical \& Health Informatics (BHI); February 16, 2017. IEEE; 133-136 
22 Báez M, Ibarra F, Far IK, Ferron M, Casati F. Online group-exercises for older adults of different physical abilities. Paper presented at: 2016 International Conference on Collaboration Technologies and Systems (CTS); October 31, 2016; IEEE; $524-533$

23 Vaira L, Bochicchio MA, Conte M, Casaluci FM, Melpignano A. MamaBot: a system based on ML and NLP for supporting women and families during pregnancy. Paper presented at: Proceedings of the 22nd International Database Engineering \& Applications Symposium; June 18, 2018; New York, NY: ACM; 273-277

$24 \mathrm{Wu}$ J, Sinnott RO, Effendy J, Glöckner S, Hu W, Li J. Usage patterns and data quality: a case study of a national type-1 diabetes study. Paper presented at: Proceedings of the 1st International Conference on Medical and Health Informatics 2017 ; May 20, 2017; New York, NY: ACM; 18-27

25 Cruciani F, Cleland I, Nugent C, McCullagh P, Synnes K, Hallberg J. Automatic annotation for human activity recognition in free living using a smartphone. Sensors (Basel) 2018; 18 (07) 2203

26 Sourial M, Elnaggar A, Reichardt D. Development of a virtual coach scenario for hand therapy using LEAP motion. Paper presented at: 2016 Future Technologies Conference (FTC); December 2016; IEEE; 1071-1078

27 Haug S, Castro RP, Filler A, Kowatsch T, Fleisch E, Schaub MP. Efficacy of an Internet and SMS-based integrated smoking cessation and alcohol intervention for smoking cessation in young people: study protocol of a two-arm cluster randomised controlled trial. BMC Public Health 2014; 14 (01) 1140

28 Shamekhi A, Trinh H, Bickmore TW. , et al. A virtual self-care coach for individuals with spinal cord injury. Paper presented at: Proceedings of the 18th International ACM SIGACCESS Conference on Computers and Accessibility; October 23, 2016; New York, NY: ACM; 327-328

29 Villalonga C, Hermens H, Herrera LJ., et al. Ontological modeling of motivational messages for physical activity coaching. Paper presented at: Proceedings of the 11th EAI International Conference on Pervasive Computing Technologies for Healthcare; May 23, 2017; New York, NY: ACM; 355-364

30 Boratto L, Carta S, Mulas F, Pilloni P. An e-coaching ecosystem: design and effectiveness analysis of the engagement of remote coaching on athletes. Pers Ubiquitous Comput 2017; 21 (04) 689-704

31 Wang Y, Fadhil A, Reiterer H. Supporting action planning for sedentary behavior change by visualizing personal mobility patterns on smartphone. Paper presented at: Proceedings of the 12th EAI International Conference on Pervasive Computing Technologies for Healthcare; May 21, 2018; New York, NY: ACM; 396-401

32 Ghose S, Barua JJ. Toward the implementation of a topic specific dialogue based natural language conversational agent as an undergraduate advisor. Paper presented at: 2013 International Conference on Informatics, Electronics \& Vision (ICIEV); May 17, 2013; IEEE; 1-5

33 Fadhil A, Villafiorita A. An adaptive learning with gamification \& conversational UIs: the rise of CiboPoliBot. Paper presented at: Adjunct Publication of the 25th Conference on user Modeling, Adaptation and Personalization; July 9, 2017; New York, NY: ACM; 408-412

34 Lokman AS, Zain JM, Komputer FS, Perisian K. Designing a conversational agent for diabetic users. Paper presented at: International Conference on Software Engineering \& Computer Systems (ICSECS'09); October 2009; 19-21

35 Sperling RA, Aisen PS, Beckett LA. , et al. Toward defining the preclinical stages of Alzheimer's disease: recommendations from the National Institute on Aging-Alzheimer's Association workgroups on diagnostic guidelines for Alzheimer's disease. Alzheimers Dement 2011; 7 (03) 280-292

36 Veen EV, Bovendeert JF, Backx FJG, Huisstede BMA. E-coaching: New future for cardiac rehabilitation? A systematic review. 2017; 100 (Suppl. 12) 2218-2230

37 Bickmore TW, Pfeifer LM, Byron D. , et al. Usability of conversational agents by patients with inadequate health literacy: evidence from two clinical trials. J Health Commun 2010; 15 (Suppl. 02) 197-210

38 Schulman D, Bickmore TW, Sidner CL. An intelligent conversational agent for promoting long-term health behavior change using motivational interviewing. Paper presented at: AAAI Spring Symposium: AI and Health Communication; March 20, 2011

39 Johnston B, Wheeler L, Deuser J, Sousa KH. Outcomes of the Kaiser Permanente tele-home health research project. Arch Fam Med 2000; 9 (01) 40-45

40 Borawska-Popielarz M. Addressing internalizing problems in middle school youth with check in/check out. Available at: https://scholarworks.waldenu.edu/cgi/viewcontent.cgi?referer=https://www.google.com/\&httpsredir=1\&article=3124\&conte $\mathrm{xt}=$ dissertations . Accessed April 15, 2019

41 Schwarzer R, Lippke S, Luszczynska A. Mechanisms of health behavior change in persons with chronic illness or disability: the Health Action Process Approach (HAPA). Rehabil Psychol 2011; 56 (03) 161-170

42 Venkatesh V, Davis FD. A theoretical extension of the technology acceptance model: four longitudinal field studies. Manage Sci 2000; 46 (02) 186-204

43 Hassenzahl M, Burmester M, Koller F. AttrakDiff: Ein Fragebogen zur Messung wahrgenommener hedonischer und pragmatischer Qualität. Paper presented at: Mensch \& Computer 2003; Teubner Verlag, Vieweg + ; 2003 :187-196

44 United States Department of Agriculture. Choose MyPlate. How many vegetables are needed daily or weekly? 2012. Available at: https://www.choosemyplate.gov/vegetables . Accessed April 15, 2019 
45 Lentferink AJ, Oldenhuis HK, de Groot M, Polstra L, Velthuijsen H, van Gemert-Pijnen JE. Key components in eHealth interventions combining self-tracking and persuasive eCoaching to promote a healthier lifestyle: a scoping review. J Med Internet Res 2017; 19 (08) e277

46 Morrison LG, Yardley L, Powell J, Michie S. What design features are used in effective e-health interventions? A review using techniques from critical interpretive synthesis. Telemed J E Health 2012; 18 (02) 137-144

47 Schwarzer R. Modeling health behavior change: how to predict and modify the adoption and maintenance of health behaviors. Appl Psychol 2008; 57 (01) 1-29

48 Cole-Lewis H, Kershaw T. Text messaging as a tool for behavior change in disease prevention and management.

Epidemiol Rev 2010; 32 (01) 56-69

49 Tatai G, Csordás A, Kiss Á, Laufer L, Szaló A. The chatbot who loved me. Paper presented at: Proceedings of the ECA Workshop of AAMAS 2003 ; Melbourne, Australia; 2003 